\title{
Traduire
}

Une autre perspective sur r tr traduction

Revue française de la traduction

$240 \mid 2019$

Quand la politique s'en mêle

\section{Finance, éthique et traduction}

\section{Pierre Jeanson}

\section{OpenEdition}

Journals

Édition électronique

URL : http://journals.openedition.org/traduire/1730

DOI : 10.4000/traduire.1730

ISSN : 2272-9992

\section{Éditeur}

Société française des traducteurs

\section{Édition imprimée}

Date de publication : 20 juin 2019

Pagination : 96-106

ISSN : 0395-773X

\section{Référence électronique}

Pierre Jeanson, «Finance, éthique et traduction», Traduire [En ligne], 240 | 2019, mis en ligne le 20 juin 2019, consulté le 06 février 2020. URL : http://journals.openedition.org/traduire/1730; DOI : 10.4000/ traduire. 1730 


\section{Finance, éthique et traduction}

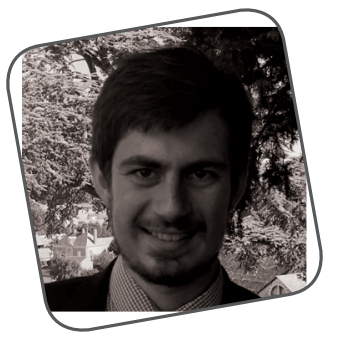

\section{Pierre Jeanson}

"Spéculation», "bulle», "subprimes», "krach boursier»: voilà ce à quoi vous pensez peut-être lorsqu'il est question de finance. Souvent, cette branche de l'économie est percue comme opaque et désincarnée. Elle ne profiterait qu'aux plus riches et jetterait les plus pauvres dans la faim et le chômage. Si la crise de 2008 nous a montré où pouvait conduire une finance dérégulée et déconnectée de l'économie réelle, peut-on parler de finance éthique? Par ailleurs, comment lier éthique et finance en traduction et dans le monde du travail en général?

En France, peut-être plus qu'ailleurs, l'argent est un sujet tabou. Les idées de gauche ainsi que la morale catholique peuvent nous inciter à nous méfier de la finance. Pourtant, le marxisme est un courant de pensée matérialiste. Et les grandes traditions religieuses ne rejettent pas l'argent en tant que tel. Après un aperçu de la finance éthique selon différentes écoles, nous verrons comment agir concrètement pour un monde plus juste, que l'on soit investisseur, traducteur ou monsieur Tout-le-Monde...

\section{Finance éthique: différentes écoles pour un même but}

Les religions et les philosophies proposent des réponses aux questions fondamentales de la vie. Que disent-elles de l'argent? 
Que l'on se rattache ou non à l'un de ces courants de pensée, comment investir concrètement de manière éthique?

Dans la religion juive, posséder des richesses n'est pas considéré comme honteux, mais comme une bénédiction divine, puisque s'enrichir permet d'avoir les moyens de donner. Au Moyen Âge, les juifs étaient parfois mal vus par les chrétiens, car ils étaient les seuls à pouvoir prêter à intérêts - pratique alors interdite par l'Église. Les banquiers juifs étaient donc nécessaires au fonctionnement de l'économie. C'est de là que vient le cliché sur les Juifs et l'argent, dont souffre toujours cette communauté aujourd'hui. Malheureusement, lorsque les débiteurs médiévaux ne pouvaient pas rembourser leurs emprunts, par exemple à cause d'une famine ou d'une épidémie, ils s'en prenaient quelquefois de manière violente à leurs créanciers.

La Bible hébraïque énonce cependant une législation sociale exigeante, qui invite à réduire les inégalités au sein de la communauté israélite, ainsi qu'entre les Juifs et les immigrés vivant sur leur territoire. D'ailleurs, de nombreux prophètes de l'Ancien Testament haranguent leur peuple au nom de Dieu afin qu'ils aient le souci des plus pauvres. En effet, selon Jacques Attali, il est attesté que le tout premier impôt sur le revenu de l'histoire de l'humanité est apparu sous le roi Salomon, au $x^{e}$ siècle avant notre ère. Avec un taux situé entre 10 et $20 \%$ des ressources de chaque contribuable, la tsedaka était intégralement redistribuée aux personnes dans le besoin. Le Talmud de Jérusalem ( $V^{e}$ siècle) et celui de Babylone ( $V^{e}$ siècle) réglementent quant à eux l'économie et la finance d'une manière très précise. Ils édictent la fixation d'un prix juste, l'interdiction de la spéculation, ainsi qu'un ensemble de normes sociales et même environnementales.

Le christianisme s'inscrit dans la continuité du judaïsme, avec la charité pour règle d'or. Dans le Nouveau Testament, il est souvent question de donner sans compter et de se détacher des choses matérielles. Dans l'Évangile de Matthieu, Jésus se montre très clair à ce sujet: "Nul ne peut servir deux maîtres: ou bien il haïra l'un et aimera l'autre, ou bien il s'attachera à l'un et méprisera l'autre. Vous ne pouvez pas servir à la fois Dieu et l'Argent'.» «Recherche du bien commun», "option préférentielle pour les 
pauvres», "destination universelle des biens»: ces concepts techniques de la pensée sociale chrétienne enseignent qu'il n'est néanmoins pas interdit d'être riche. Mais ces richesses doivent être acquises honnêtement et servir la construction d'un monde plus humain et plus juste. Le chrétien n'est donc pas le propriétaire de ses biens, mais il en est le gérant.

Selon Max Weber, l'éthique protestante, qui voit dans l'oisiveté un très grand péché, serait à l'origine du capitalisme. D'après le sociologue, de tout temps, des personnes ont cherché à faire le lien entre les convictions religieuses, l'éthique et l'action. Dans ses écrits, il expose son intuition que les croyances poussent à agir. Dans cette perspective, la bénédiction de Dieu sur l'homme lui donne le goût de "s'enrichir», car la richesse est signe de l'abondance de Dieu.

La Congrégation pour la doctrine de la foi et le dicastère pour le service du développement intégral (deux organes de l'administration centrale du Vatican) ont publié en janvier 2018 un document sur l'éthique financière intitulé Oeconomicae et pecuniariae quaestiones. L'idée première est de mettre l'homme au centre de l'économie. En effet, le système économique n'est pas appréhendé uniquement d'un point de vue matériel. Il est aussi un ensemble de relations entre des personnes qui doivent être respectées dans leur intégralité. Ce document condamne la spéculation et invite à repenser le système financier afin qu'il soit au service de l'économie réelle. Cela permettrait, selon le Saint-Siège, d'éviter de nouvelles crises financières et d'aider les plus défavorisés à sortir de la misère. Le texte dénonce également les inégalités entre les experts de la finance et l'investisseur lambda. Ce dernier peut facilement se faire escroquer en plaçant son argent dans des instruments trop complexes dont il ne comprend pas toutes les subtilités. Enfin, l'évasion fiscale, la corruption, le surendettement des États et le blanchiment d'argent sont dénoncés. Tous ces phénomènes, jugés immoraux, seraient la cause profonde des dysfonctionnements du système économique. Oeconomicae et pecuniariae quaestiones propose quelques solutions:

- une séparation nette des pouvoirs politiques, économiques et financiers, pour rendre sa souveraineté au peuple;

- une transparence accrue, pour permettre à chaque investisseur de savoir où est placé son argent;

- une meilleure responsabilité sociale des entreprises (RSE); 
- une plus grande diversité économique et financière, ainsi que des fonds propres plus conséquents pour les banques, afin que le système soit plus résistant en cas de crise;

- une réglementation renforcée;

- la présence d'un comité d'éthique au sein des banques;

- une taxe sur les transactions offshore, inspirée de la fameuse taxe Tobin, afin de verser l'argent collecté aux organismes compétents en matière d'éradication de la faim dans le monde;

- une réduction de la bureaucratie de nos administrations, pour lutter contre l'endettement des États qui pèse sur le contribuable.

Mais au-delà de ces propositions politiques, Oeconomicae et pecuniariae quaestiones rappelle que chaque consommateur peut agir à son échelle pour que le monde soit plus humain et plus juste, notamment en orientant ses achats et ses placements.

Sur ce point, les agissements d'une partie de l'Église catholique n'ont pas toujours été cohérents. En effet, des liens ont longtemps uni la mafia à certaines paroisses du sud de l'Italie. De l'argent sale a même été déposé à la banque du Vatican. Cela a commencé au moment de la guerre froide, lorsque des mafieux ont vu l'avantage qu'ils pourraient tirer d'une alliance avec l'Église et certains politiciens contre les communistes, alors très populaires en Sicile. Mais sur le terrain, des prêtres courageux ont dénoncé le crime organisé, au péril de leur vie. En 1993, Jean-Paul II s'est clairement opposé à la mafia en rappelant le commandement "Tu ne tueras point». En juin 2014, le pape François est allé plus loin en excommuniant les mafieux. Un an auparavant, il avait béatifié et reconnu comme martyr le père Giuseppe Puglisi, assassiné en 1993 pour s'être opposé aux gangsters de Palerme. Quant à la banque du Vatican, elle a subi une opération "mains propres», déjà amorcée sous Benoît XVI. Plusieurs milliers de comptes liés au blanchiment d'argent ont ainsi été clôturés.

Dans l'islam, la finance se base sur cinq piliers:

- le refus catégorique de l'usure (il est interdit de prêter ou d'emprunter avec des intérêts);

- l'interdiction de spéculer et de se livrer à des jeux de hasard;

- l'interdiction de faire commerce de biens «illicites» (haram en arabe), notamment d'alcool, de drogue, de tabac et de pornographie; 
- l'obligation de partager les profits et les pertes entre la personne qui apporte le capital et celle qui l'exploite (le bon sens invite donc à ne pas financer des entreprises ou des ménages trop endettés, ce qui évite de déclencher des crises comme celle des subprimes);

- l'obligation d'adosser les titres à des actifs tangibles, afin de rester connecté à l'économie réelle.

Le fait que la finance islamique fonctionne différemment de la finance occidentale a relativement bien préservé le monde musulman de la crise de 2008. Notons par ailleurs que Muhammad Yunus a inventé le microcrédit en conformité avec l'éthique musulmane en matière d'argent. Ce professeur d'économie a fondé en 1976 la Grameen Bank pour les populations marginalisées du Bangladesh, développant ainsi un système de microprêts qui permet aujourd'hui à de nombreuses personnes dans le monde entier de sortir de la misère. En 2006, il a reçu le prix Nobel de la paix.

En revanche, d'autres banques aux valeurs prétendument islamiques pratiquent une usure qui ne dit pas son nom. Elles escroquent ainsi des musulmans qui veulent emprunter en respectant la charia. Leurs clients payent finalement des intérêts beaucoup plus importants que s'ils avaient emprunté auprès d'une banque classique.

Par ailleurs, l'aumône (zakât en arabe) est le troisième des cina piliers de l'islam. Elle sert à la fois à assurer la solidarité avec les croyants les plus démunis et à aider les fidèles donateurs à se détacher des biens matériels. C'est pour cette raison que beaucoup de pays musulmans n'ont pas mis en place de système d'imposition étatique. Les musulmans doivent, par la zakât, redistribuer les biens qu'ils possèdent.

Enfin, le point de vue des différentes écoles bouddhiques peut être résumé dans cette citation du maître tibétain Tulku Thondup: "Non pas que gagner de l'argent constitue en soi une cause de souffrance, mais livrer sa vie à la tyrannie de possessions qui n'ont jamais de fin porte un coup fatal à la paix et à la joie.» Là aussi, il s'agit de vivre dans un réel détachement conduisant au bonheur.

Remarquons que le judaïsme, le christianisme, l'islam et le bouddhisme ont en commun de condamner - ou de ne pas recommander - les jeux d'argent et de hasard.

Cet aperçu nous enseigne donc que, dans ces quatre traditions religieuses, ce n'est pas l'argent qui est bon ou mauvais, mais 
l'usage qui en est fait. Bien évidemment, ces valeurs sont aussi partagées par de nombreuses personnes qui se rattachent à d'autres convictions, qu'elles soient religieuses ou non. Ce regard commun considère donc que la finance est légitime sur le plan éthique si elle est utilisée non pas pour s'enrichir à tout prix, mais pour créer de l'emploi, dans le respect de l'homme et de son environnement.

Aujourd'hui, il est tout à fait possible de placer son argent dans des fonds ou des sociétés qui respectent certains critères environnementaux, sociaux et de gouvernance (ESG). On parle alors d'investissement socialement responsable (ISR), ou d'investissement responsable (IR). Lorsque I'IR/ISR vise à réduire le réchauffement climatique, on parle de "finance verte». Par exemple, les obligations vertes (green bonds) correspondent à des prêts aux entreprises qui développent les énergies renouvelables. En France, le label public qui fait foi en la matière est TEEC (pour «Transition énergétique et écologique pour le climat»). Il est également possible de placer son argent conformément à d'autres valeurs, par exemple dans des fonds qui investissent à l'échelle locale afin d'y maintenir ou d'y créer de l'emploi. D'ailleurs, les fonds d'investissement de proximité (FIP), qui investissent dans des PME locales, sont avantageux sur le plan fiscal; en revanche, ce sont des placements risqués. Enfin, certains fonds investissent exclusivement dans des entreprises qui respectent les valeurs d'une philosophie ou d'une religion particulière. Par exemple, certains organismes de placement collectif (OPC) gérés par Meeschaert Asset Management s'engagent à respecter au mieux la pensée sociale chrétienne. Cela inclut, notamment, en plus des critères ESG classiques, le refus d'investir dans la pornographie. De même, il existe des fonds conformes aux règles de la finance islamique pour les personnes désirant respecter les principes de la charia.

Par ailleurs, les placements éthiques entendent occasionner une certaine rentabilité. En témoigne la performance de la SICAV Sycomore Happy@Work. Ce compartiment ISR créé en 2015 privilégie l'investissement dans des entreprises qui s'engagent pour le bonheur au travail de leurs salariés; celui-ci rejaillissant très positivement sur la créativité, la productivité et donc la rentabilité, la performance du fonds a presque toujours été largement au-dessus de celle de son indice de référence, EURO STOXX TR. 
Mais comme le souligne Thibault Leroux, journaliste à Amnesty International, l'investissement socialement responsable vise avant tout le profit. Certains fonds peuvent donc être moyennement regardants sur tel ou tel critère de sélection. La finance éthique propose en fait une autre option, moins lucrative, mais souvent plus intègre, à savoir l'épargne solidaire. Celle-ci met l'argent des épargnants au service de l'économie sociale et solidaire (ESS) ou de diverses associations humanitaires ou caritatives. Certaines d'entre elles proposent aux particuliers de placer leur argent dans des fonds (souvent labellisés ISR) tout en acceptant que 25 à $100 \%$ des gains soient reversés à ces organismes sans but lucratif. C'est le système des "produits de partage». Il est utilisé, entre autres, par Habitat et Humanisme, le CCFD-Terre Solidaire et Amnesty International.

En somme, finance et éthique sont tout à fait compatibles. Nous avons même l'embarras du choix pour placer notre argent au service du bien commun.

\section{Et la traduction dans tout ça?}

Lorsque j'ai commencé mes études de traduction économique et juridique à l'université de Cergy-Pontoise, je n'envisageais pas de me spécialiser en traduction financière. En effet, ce domaine me paraissait si opaque et technique que je ne me sentais pas à la hauteur. De plus, façonné de valeurs me poussant à être naturellement méfiant à l'égard de l'argent, je ne voyais pas quel était le sens d'une telle activité. Aussi ai-je hésité avant d'accepter un stage de fin d'études dans ce domaine de spécialité.

Finalement, j'ai rapidement compris que cette expérience professionnelle pouvait avoir une visée éthique intéressante. En effet, à la suite de l'éclatement de la bulle financière en 2008 , les responsables politiques se sont rendu compte que la finance devait être mieux encadrée. Ainsi, une série de directives européennes limite-t-elle désormais le pouvoir des institutions financières, les obligeant notamment à faire preuve d'une transparence absolue afin de réduire ces prises de risques. C'est en particulier le cas de la directive Markets in Financial Instruments Directive (MiFID). De ce fait, ces établissements publient de nombreux documents (prospectus de vente et documents d'information clés pour l'investisseur) afin d'informer les investisseurs et d'encadrer leurs placements. Et comme les flux de capitaux circulent 
quotidiennement à l'échelle internationale, cette documentation doit être traduite rapidement. C'est dans cette optique qu'a été fondée la société FinTech; elle crée des moteurs de traduction automatique dans le domaine financier, en vue de permettre la transparence à l'échelle mondiale. En acceptant un stage de six mois au sein de cette structure, j'allais donc apporter ma pierre à la moralisation de la finance.

Pourtant, évoquer la traduction automatique (TA) soulève un autre problème éthique: la perte d'emploi due au remplacement de l'homme par la machine. Néanmoins, les promoteurs de la TA considèrent que proposer une traduction à moindre coût permet de traduire des documents qui n'auraient jamais été traités manuellement, faute de budget. Mais l'intelligence artificielle ne s'adaptera jamais aussi rapidement que le cerveau humain et le travail d'une personne en chair et en os reste indispensable:

- pour créer le moteur de traduction en traitant des bases de données linguistiques (cela équivaut à un exercice de révision de textes déjà traduits);

- pour relire la traduction nécessairement imparfaite de la machine (ce que le jargon appelle la «post-édition»).

Ses défenseurs estiment en effet que la traduction automatique ne menace pas l'emploi des traducteurs professionnels. Pour autant, même dans le cas où les traducteurs garderaient leur emploi, une autre question éthique se pose. En effet, quelle satisfaction intellectuelle retire-t-on du nettoyage de mémoires de traduction ou de la post-édition, comparé à la tâche gratifiante et épanovissante qu'est la traduction?

Malheureusement, d'autres chercheurs sont beaucoup moins optimistes. Avec l'arrivée de la TA neuronale, ils estiment que certains emplois des traducteurs sont réellement menacés, notamment dans les combinaisons de langues les plus courantes et les spécialités les moins créatives. Peut-on déjà voir dans la baisse progressive des tarifs une marque de ce phénomène?

Comme toute compétence, la traduction financière - et la traduction en général - peut être utilisée à des fins douteuses. En effet, depuis que je suis installé en freelance, on m'a déjà proposé de traduire pour une cause qui allait à l'encontre de mon éthique personnelle. J'ai donc refusé ce projet qui me paraissait vide de sens. Les études prouvent d'ailleurs que le bonheur au travail est en partie lié à la conformité de notre vie professionnelle avec 
nos valeurs. Un auditeur financier senior spécialisé dans les comptes d'organismes à but non lucratif m'a assuré qu'il ne pourrait pas exercer son métier si ses clients étaient des entreprises à l'éthique professionnelle douteuse.

II m'est même arrivé de refuser un très gros projet de traduction grassement rémunéré, car le donneur d'ordre était une secte. J'aurais pu m'enrichir, mais j'aurais été payé avec de l'argent volé à des personnes endoctrinées, et mon travail aurait servi à en endoctriner d'autres. En revanche, si j'ai choisi de ne pas contribuer à cette cause qui me paraissait injuste, je suis heureux de traduire aujourd'hui pour des entreprises et des associations qui agissent conformément à mes valeurs.

Car rester intègre n'est pas nécessairement néfaste à la rentabilité. Les entreprises qui verdissent leur gestion, et donc leur image, jouissent d'une bonne réputation et bien souvent d'une excellente santé financière. Selon une étude de France Stratégie relayée par la plateforme E-RSE, mettre en œuvre la responsabilité sociale des entreprises accroit la performance de $13 \%$. À l'inverse, un scandale bien relayé sur le plan médiatique peut faire baisser le chiffre d'affaires de manière significative. Quant au bonheur au travail, il ferait progresser la productivité de $12 \%$, d'après une enquête réalisée par l'université de Warwick en 2014.

Comment appliquer alors la RSE et verdir son image lorsqu'on est traducteur? Travailler seul devant son ordinateur implique une empreinte carbone et une gestion des ressources humaines très limitées. À nous d'être inventifs pour aller plus loin en travaillant de manière éthique, au-delà du code de déontologie de la SFT!

Du respect des tarifs auprès des sous-traitants à la pratique du mécénat de compétences au sein d'un organisme à but non lucratif, en passant par le versement d'une partie des bénéfices à une association, les exemples sont légion. En outre, la traduction et la relecture peuvent être exercées auprès d'une œuvre humanitaire ou caritative, et certains publics défavorisés sont à la recherche de cours de français, de langues vivantes et d'autres disciplines. Enfin, et notamment dans le contexte de réchauffement climatique que nous connaissons, nous ne dirons jamais assez l'importance d'un mode vie écologique qui contribue à réduire notre empreinte carbone (moyens de transport, alimentation, énergie, papier, etc.). 
Si chaque traducteur est mû par des valeurs qui lui sont propres, comment communiquer sur ce sujet pour attirer des clients? Dans sa théorie dite du «cercle d'or», Simon Sinek a prouvé que l'ordre de communication marketing le plus vendeur n'est pas «Quoi? Comment? Pourquoi?»-ce que nous avons tous tendance à faire instinctivement; mais qu'il faut au contraire penser: "Pourquoi? Comment? Quoi?» En d'autres termes, nos valeurs feront davantage vibrer le prospect que ce que l'on vend et comment on le fabrique. C'est ainsi qu'ont raisonné les plus grands leaders, de Martin Luther King à Steve Jobs. Ainsi, mettre en avant nos valeurs sur notre site professionnel peut être décisif pour qu'un prospect nous contacte. Bien sûr, dire que nous faisons du vélo ou que nous utilisons des ampoules basse consommation peut faire sourire les internautes. Mais nous pouvons tout simplement dire que nous cherchons à réduire notre empreinte carbone. Cela peut inciter les prospects à nous poser des questions auxquelles nous répondrons dans l'échange qui suivra. En revanche, les actions concrètes sur le plan social peuvent être communiquées de manière plus précise. Nous pouvons même nommer les associations que nous soutenons ou dans lesquelles nous sommes engagé.e.s. Il faut néanmoins solliciter leur accord et s'assurer qu'elles ne sont pas trop marquées politiquement ou religieusement.

En finance comme en traduction, et dans le monde professionnel en général, l'éthique revêt donc une dimension primordiale. Elle permet de faire sens, d'avoir un impact positif sur les plans social et environnemental, ainsi que d'être plus heureux au travail. D'ailleurs, si l'on en croit Aristote, la recherche du bonheur n'est-elle pas au fondement de toute morale?

\section{pierrejeanson.trad@outlook.fr avec le concours de Jordana Do Rosário pour la relecture}

Installé à Angers, Pierre Jeanson (www.pierre-jeanson.fr) est traducteur indépendant depuis 2016. II est spécialisé en traduction juridique et financière, mais traduit aussi des documents à thème religieux. Ses langues de travail sont le français, l'espagnol, l'anglais et, dans une moindre mesure, le portugais. 
Association épiscopale liturgique francophone (AELF), Évangile de Jésus-Christ selon saint Matthieu, Paris, Association épiscopale liturgique francophone (AELF), 2013, www.aelf.org/ bible/Mt/6, consulté le 13 mai 2019.

ATTALI Jacques et CONAN Éric, Les juifs, les chrétiens et l'argent, L'Express, 2002, www.lexpress.fr/informations/les-juifs-les-chretiens-et-l-argent_646897.html, consulté le 13 mai 2019.

BALAY Raphaël, Simon Sinek: Comment les grands leaders inspirent l'action?, Oser entreprendre, 2012, www.oser-entreprendre.fr/tags/cercle-dor/, consulté le 13 mai 2019.

CHATELAIN Gaël, Si votre entreprise appliquait ces 10 conseils, vous seriez plus heureux au bureau, LinkedIn, 2016, www.linkedin.com/pulse/10-trucs-simples-pour-am\% $\mathrm{C}_{3} \%$ A9liorer-lambiance-dans-une-\%C3\%A9quipe-chatelain, consulté le 13 mai 2019.

Congrégation pour la doctrine de la foi \& Dicastère pour le service du développement intégral, Oeconomicae et pecuniariae quaestiones: Considérations pour un discernement éthique sur certains aspects du système économique et financier actuel, Cité du Vatican, Congrégation pour la doctrine de la foi \& dicastère pour le service du développement intégral, 2018, www.vatican. va/roman_curia/congregations/cfaith/documents/rc_con_cfaith_doc_20180106_oeconomicae-et-pecuniariae_fr.html\#_ftnref48, consulté le 13 mai 2019.

Globislam, Les 5 piliers de la finance islamique, France, OverBlog, http://globislam.over-blog. com/article-les-5-piliers-de-la-finance-islamique-84886097.html, consulté le 13 mai 2019.

LEROUX Thibault, Finance éthique: des valeurs gagnantes, Amnesty International, 2018, www.amnesty.fr/actualites/finance-ethique--des-valeurs-gagnantes?utm_source=newshebdo\&utm_ medium=email\&utm_campaign=news-hebdo-2018-01-19, consulté le 13 mai 2019.

VANIER Jean, Le Goût du bonheur au fondement de la morale avec Aristote, Paris, Éditions France Loisirs, coll. «Philanthrop'», 2007. 